\title{
Consensus report of the European Federation of Conservative Dentistry: erosive tooth wear-diagnosis and management
}

\author{
T. S. Carvalho ${ }^{1}$ - P. Colon ${ }^{2}$ C. Ganss ${ }^{3}$ - M. C. Huysmans ${ }^{4}$ - A. Lussi ${ }^{1}$ - N. Schlueter ${ }^{3}$. \\ G. Schmalz ${ }^{5,1}$ - R. P. Shellis ${ }^{1}$ A. B. Tveit ${ }^{6}$ A. Wiegand ${ }^{7}$
}

Received: 1 June 2015 / Accepted: 9 June 2015 / Published online: 1 July 2015

(C) Springer-Verlag Berlin Heidelberg 2015

\begin{abstract}
Objective Due to an increased focus on erosive tooth wear (ETW), the European Federation of Conservative Dentistry (EFCD) considered ETW as a relevant topic for generating this consensus report.

Materials and methods This report is based on a compilation of the scientific literature, an expert conference, and the approval by the General Assembly of EFCD.

Results ETW is a chemical-mechanical process resulting in a cumulative loss of hard dental tissue not caused by bacteria, and it is characterized by loss of the natural surface morphology and contour of the teeth. A suitable index for classification of ETW is the basic erosive wear examination (BEWE). Regarding the etiology, patient-related factors include the predisposition to erosion, reflux, vomiting, drinking and eating habits, as well as medications and dietary supplements. Nutritional factors relate to the composition of foods and beverages, e.g., with low $\mathrm{pH}$ and high buffer capacity (major risk factors),
\end{abstract}

All authors contributed equally to this work. Names merely appear in alphabetical order.

A. Lussi

adrian.lussi@zmk.unibe.ch

1 Department of Preventive, Restorative and Pediatric Dentistry, University of Bern, Freiburgstrasse 7, CH-3010 Bern, Switzerland

2 Hôpital Rothschild, AP-HP, Service d'Odontologie, Université Paris Diderot, Paris, France

3 Department of Conservative and Preventive Dentistry, Dental Clinic, Justus Liebig University Giessen, Schlangenzahl 14,

DE-35392 Giessen, Germany and calcium concentration (major protective factor). Occupational factors are exposition of workers to acidic liquids or vapors. Preventive management of ETW aims at reducing or stopping the progression of the lesions. Restorative management aims at reducing symptoms of pain and dentine hypersensitivity, or to restore esthetic and function, but it should only be used in conjunction with preventive strategies.

Conclusions Effective management of ETW includes screening for early signs of ETW and evaluating all etiological factors.

Clinical Relevance ETW is a clinical condition, which calls for the increased attention of the dental community and is a challenge for the cooperation with other medical specialities.

Keywords Dental erosion · Diagnosis · Etiology $\cdot$ Preventive measures $\cdot$ Restorative therapy
4 College of Dental Sciences, Radboud University Medical Center, Radboud University Nijmegen, PO Box 9101, NL-6500 HB Nijmegen, The Netherlands

5 Department of Operative Dentistry and Periodontology, University Medical Centre Regensburg, Franz-Josef-Strauss-Allee 11, DE-93042 Regensburg, Germany

6 Department of Cariology and Gerodontology, Institute of Clinical Dentistry, Faculty of Dentistry, University of Oslo, NO-0317 Oslo, Norway

7 Department of Preventive Dentistry, Periodontology and Cariology, Georg August University Göttingen, Robert-Koch-Strasse 40, DE-35075 Göttingen, Germany 


\section{Aim of the consensus report}

This consensus report of the European Federation of Conservative Dentistry (EFCD) is intended to assist practitioners with diagnosis and decision-making about erosive tooth wear (ETW). The recommendations in this document are not intended to define a standard of care but rather should be integrated with a practitioner's professional evaluation and judgment, as well as a patient's needs and preferences [1].

Over the recent decades, there has been increased focus on ETW. The prevalence has been studied all over the world and the results varied widely. In many regions, including Europe, the prevalence of the condition is high [2]. For instance, a recent European study indicated that around $30 \%$ of the population attending general practices aged 18-35 years has at least one tooth with advanced erosive tooth wear [3]. Therefore, the EFCD considered this topic as relevant to daily dental practice.

To generate this report, the Board of the EFCD appointed an editorial group to produce a text proposal. This text proposal was based on a series of literature reviews compiled in a recent monograph [4]. The proposal was then meticulously discussed in a consensus conference in Bern, Switzerland, on the 29th and 30th of April, 2015, during which the report was written. The report has since been approved by the EFCD Board and the general assembly of the EFCD on the 15th of May, 2015.

\section{Definition of erosive tooth wear}

Erosive tooth wear is defined as a chemical-mechanical process resulting in a cumulative loss of hard dental tissue not caused by bacteria.

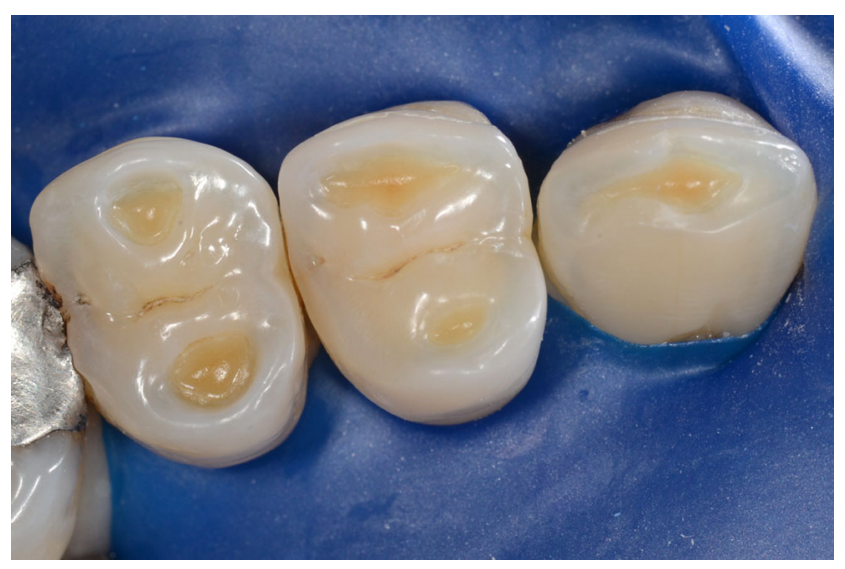

Fig. 1 Erosive tooth wear on occlusal surfaces of premolars, with deep cupping of the cusps and flattening of the occlusal structures (BEWE 2advanced stage)

\section{Clinical assessment and classification}

During a lifetime, teeth are exposed to a number of physical and chemical insults, which, to a various extent, contribute to the wear of dental hard tissues. Although all these factors act together, the morphology of defects may vary depending on the predominant cause.

ETW is also multifactorial with acid as the main cause. It ceases from progression if these impacts can be controlled. The assessment therefore consists of the differential diagnosis of several forms of tooth wear and the identification of all relevant causative factors. The clinical examination should be linked to a thorough history taking.

In general, ETW is characterized by loss of the natural surface morphology and contour. Typical signs of ETW on occlusal surfaces are cupping of the cusps and flattening of the occlusal structures. In advanced stages, the whole occlusal morphology can disappear and hollowed out surfaces can develop. Restorations may stand proud of the adjacent tooth surface (Figs. 1 and 2). Typical signs of ETW on smooth surfaces are flattening of the surface, and an intact rim along the gingival margin may be present. Concavities may become present, which are normally more wide than deep (Figs. 3 and 4). In all cases, these lesions occur on both permanent and deciduous teeth and can extend into dentine. Lesions can be localized (on single tooth, vestibular/oral), generalized or asymmetric, depending on the etiology.

Occlusal lesions must be distinguished mainly from attrition. Attrition is caused by the action of antagonistic teeth (e.g., grinding) and leads to matching facets; lesions typically are flat, sharp bordered, and glossy. Smooth surface lesions must be mainly distinguished from abrasion and wedge-shaped defects. Wedge-

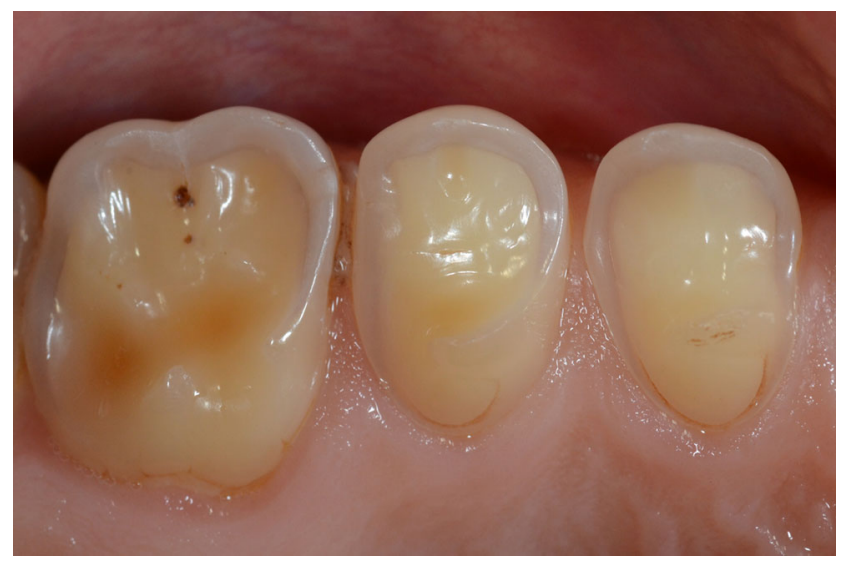

Fig. 2 Erosive tooth wear on occlusal surfaces of molar and premolars, with advanced destruction of the occlusal morphology and flattened, hollowed out surfaces (BEWE 3) 


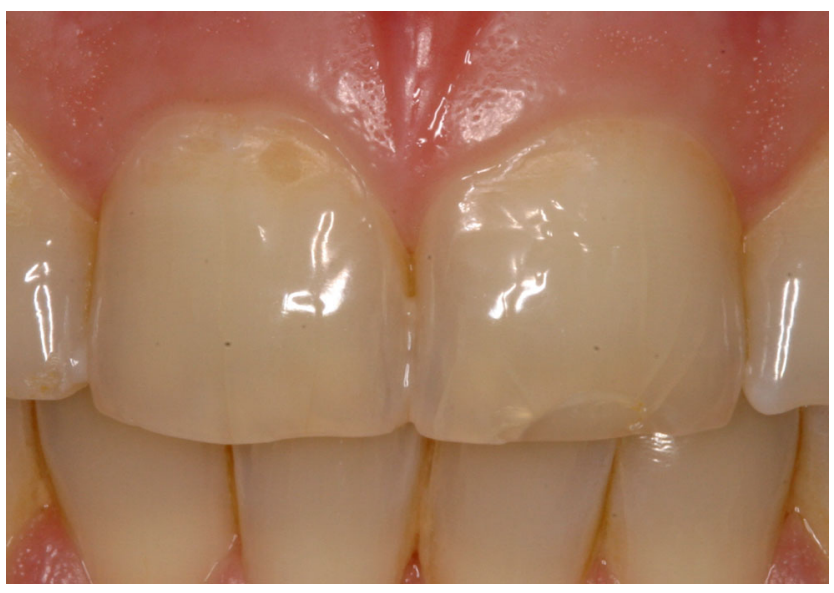

Fig. 3 Erosive tooth wear on labial surfaces of incisors, presenting small concavities and an intact rim along the gingival margin (BEWE 2)

shaped lesions are located at the cervical margin. The coronal part typically has sharp margins and cuts at right angle into the enamel surface. The apical part bottoms out to the root surface. Abrasion is predominantly caused by traumatic oral hygiene habits and may vary in appearance depending on the causative impact. The main difficulty in the clinical assessment is the interaction of the various causative factors, especially when the respective injuries act for a long time.

The assessment of progression is important, as it determines whether preventive measures are necessary or interventions implemented were successful, and may help with decision-making with respect to when and how to restore worn teeth. Clinical signs of progression are dentine hypersensitivity and dull, frosty appearance, as well as absence of staining of the lesion. Monitoring is possible with study models, standardized photographs, or with a finely graded clinical index at a tooth level.

Erosive tooth wear is, in part, a normal wear process occurring over lifetime. For the management of the condition, it is therefore necessary to distinguish between pathological and physiological loss of tooth tissues:

"Tooth wear can be regarded as pathological if the teeth become so worn that they do not function effectively or seriously mar the appearance before they are lost for other causes or the patient dies. The distinction of acceptable and pathological wear at a given age is based upon the prediction of whether the tooth will survive the rate of wear" [5].

A suitable index for classification is the Basic Erosive Wear Examination (BEWE; Table 1), which links the grading of lesions with clinical management [6]. For scoring, the dentition is divided into sextants. The most severe lesion in each sextant is recorded, and an overall score for a patient is calculated by adding the scores of all sextants.

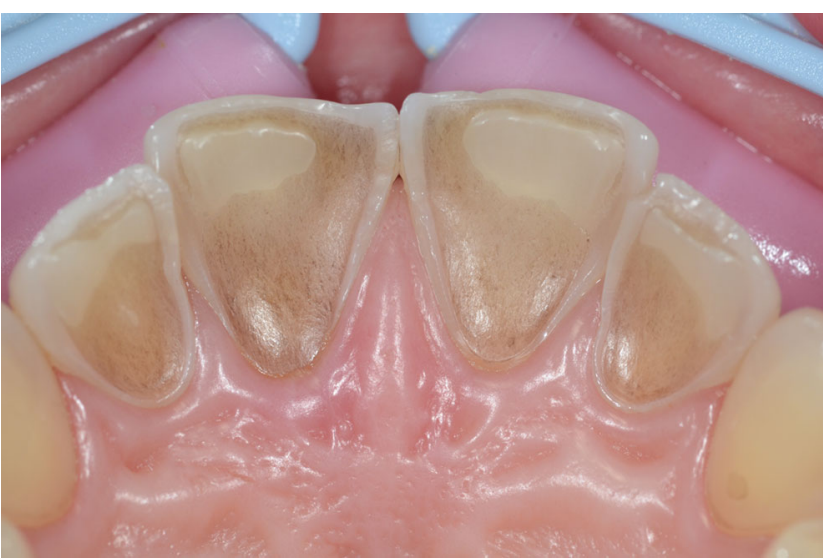

Fig. 4 Erosive tooth wear on palatal surfaces of incisors, with some enamel islands still present and an intact enamel rim observed only along the gingival margin of the lateral incisors (BEWE 3)

\section{Etiology}

The first step of ETW is softening of the surface by erosive substances of intrinsic or extrinsic origin or a combination of both. Not all acidic products are necessarily erosive, as explained below. It must be appreciated that, for a risk to be clinically significant, the exposure needs to be frequent, severe, and/or over a long period. We can distinguish between patient-related, nutritional, and occupational factors.

\section{Patient-related factors}

\section{Pre-disposition to erosion}

There is considerable variation between people in their susceptibility to erosive wear, as measured under standard conditions. Current knowledge suggests that this variation is due to differences in susceptibility of dental hard tissues to dissolution and to differences in saliva.

Saliva is protective against ETW in several ways. Saliva dilutes acidic substances and removes them from the mouth. It buffers and neutralizes acidic products, so shortens erosive episodes. Finally, salivary proteins are the source of the acquired salivary pellicle, which reduces acid erosion. Therefore, reduced salivary flow rate is a risk factor for ETW. Flow rate can be reduced by, for instance high levels of exercise, systemic disease (e.g., Sjögren's syndrome), and certain medications (e.g., antihypertensives, antidepressives).

\section{Reflux, vomiting}

Because the stomach is a source of intrinsic acid, regurgitation of stomach contents into the mouth is a cause of ETW. For the risk of ETW to be significant, frequent regurgitation over an extended period of time is necessary. Therefore, vomiting due, 
Table 1 Criteria for grading erosive wear

\begin{tabular}{ll}
\hline Score & Criteria \\
\hline Score 0 & No erosive tooth wear \\
Score 1 & Initial loss of surface texture \\
Score $2^{\text {a }}$ & Distinct defect; hard tissue loss involving $<50 \%$ of the surface area \\
Score $3^{\mathrm{b}}$ & Hard tissue loss involving $\geq 50 \%$ of the surface area \\
\hline
\end{tabular}

${ }^{a}$ In scores 2 and 3, dentine is often involved for instance, to occasional stomach disorders or to morning sickness in pregnancy is not considered a cause for concern. A raised prevalence of ETW has been identified in groups of people in which frequent and persistent regurgitation is a symptom of an underlying medical condition. Such conditions include gastro-esophageal reflux disorder $\left(\mathrm{GERD}^{1}\right)$, rumination, and eating disorders with frequent vomiting (e.g., bulimia nervosa).

\section{Drinking and eating habits}

A large proportion of erosive challenges is caused by extrinsic dietary acids (food and drink). The greater the amount of erosive products consumed per day, and the more often they are consumed, the greater the risk of ETW. Drinking habits are important; the hazard presented by an erosive acidic drink is less if it is swallowed in big gulps in a shorter period than if it is sipped over an extended period; retention of an acidic drink in the mouth before swallowing increases the hazard, especially if it is "swished" around the teeth. Clinical observations suggest that drinking through a straw positioned palatally can reduce the risk of erosion, but if acidic drinks are habitually consumed through a straw or "pull-out" drinking cap positioned in front of the teeth, the incisors can be eroded rapidly.

\section{Medications and dietary supplements}

Some medications (e.g., acidic saliva stimulants or preparations containing acetylsalicylic acid) and dietary supplements (e.g., vitamin $\mathrm{C}$ tablets) are potentially erosive if they are in the form of chewable tablets or effervescent drinks. Other medications have the side effect of reducing salivary flow, which could indirectly enhance ETW from other agents.

\section{Oral hygiene}

Normal tooth brushing has no harmful effect on sound dental tissues. The softened tooth surfaces caused by exposure to acidic products are vulnerable to tooth brushing. However, softened enamel is not remineralized by saliva over short time

\footnotetext{
$\overline{{ }^{1} \mathrm{GERD}=\mathrm{GORD}}$
}

periods, so will be worn away even in the absence of tooth brushing. Therefore, postponing brushing after eating acidic products is not a useful preventive measure, especially as brushing is a vehicle for delivery of fluoride in the prevention of caries.

\section{Nutritional factors}

The composition of foods and beverages determines how erosive they are. Analyses of many products have identified a low $\mathrm{pH}$ and a high buffer capacity as the major risk factors, and the calcium concentration as the major protective factor in determining the erosive potential. It is important not to judge erosive potential by the $\mathrm{pH}$ alone; there is no single "critical $\mathrm{pH}$ " for erosion. For instance, the $\mathrm{pH}$ of yoghurts is about 4.0, yet they are not erosive because they have high calcium concentrations. The buffer capacity of the product (determined by the type of acid and the $\mathrm{pH}$ ) affects the resistance of the product to being neutralized by saliva. If the product contains substances, such as gums, which adhere to tooth surfaces, they may retain the product at the tooth surface and hence prolong the erosive challenge. Studies have shown that milk and yoghurt consumption is related to a lower prevalence of ETW [7].

\section{Occupational factors}

In some occupations, such as wine tasting or battery manufacture, workers are exposed to acidic liquids or vapors, which can cause rapid erosion of workers' teeth. This is a rare cause of ETW.

\section{Management of erosive tooth wear}

\section{Preventive management}

The objective of preventive management of ETW is to reduce or stop progression of the lesions.

A prerequisite for effective management is to include screening for early signs of ETW in each clinical examination. When ETW has been identified, all possible etiological factors 
should be evaluated, for instance through precise questioning by the dentist, including general medical conditions, oral hygiene habits, pain, and functional problems. The patient should be instructed to record a food diary for a distinct period of time (e. g., 4 days), including specific eating and drinking habits. Where applicable, salivary flow rates may be determined. Information on clinical presentation and etiological factors should be analyzed carefully. Based on these analyses, an individually tailored preventive program should be suggested to a patient.

Intrinsic acid sources are an important cause of ETW. The dentist may play an important role in detecting relevant disorders, such as eating disorders and GERD. Signs and symptoms of GERD may be heartburn, coughing, hoarseness, and dysphagia. When intrinsic acid sources are suspected to be the main causal factor, then referral to a specialist or a general practitioner is advised.

If the food diary suggests that extrinsic erosive sources are an important causal factor, then the following advice should be given:

1. Reduce the frequency of consumption of the identified erosive foods and beverages.

2. Avoid eating and drinking habits that extend the contact time of the acid with the teeth.

3. Choose safer food alternatives, such as calcium-enriched (sports) drinks and foods, or water and milk products.

The evidence linking tooth brushing habits with ETW is limited and contradictory. Therefore, the general advice for caries management should not be modified.

Additional to causal management options, it is possible to use specific protective products or materials. Products (e.g., toothpastes or mouth rinses) containing stannous fluoride or stannous chloride have the potential for slowing the progression of ETW. For other products, data so far are sparse. If dentin hypersensitivity and pain are present as symptoms of erosive wear, the first aim of treatment should be directed at managing etiological factors. As an adjunct treatment, products against dentin hypersensitivity may be advised. Application of a layer of resin sealant or bonding material has been shown to reduce ETW progression and pain for a limited time period.

\section{Restorative management}

The objective of restorative management of ETW is to reduce or stop progression of the advanced lesion, to reduce symptoms of pain and dentine hypersensitivity, or to restore esthetic and function. It should only be preceded by or used in conjunction with preventive strategies.

As a rule, one should use the least invasive therapy. Restorative therapies comprise the use of direct (e.g., composites) or indirect (e.g., composites, ceramics, metals) restorations. There is no evidence that ETW should be treated differently from established procedures; loss of tooth tissue should be restored according to the site and extent of loss, regardless of the cause. Generally, direct procedures are less invasive than indirect, and composites have been recommended. Monitoring and evaluation of ETW management should be performed regularly during recall sessions.

Acknowledgments The consensus conference was funded by EFCD and the Department of Preventive, Restorative, and Pediatric Dentistry, University of Bern, Switzerland.

\section{References}

1. Weyant RJ, Tracy SL, Anselmo TT, Beltrán-Aguilar ED, Donly KJ, Frese WA, Hujoel PP, Iafolla T, Kohn W, Kumar J, Levy SM, Tinanoff N, Wright JT, Zero D, Aravamudhan K, Frantsve-Hawley J, Meyer DM, American Dental Association Council on Scientific Affairs Expert Panel on Topical Fluoride Caries Preventive Agents (2013) Topical fluoride for caries prevention: executive summary of the updated clinical recommendations and supporting systematic review. J Am Dent Assoc 144:1279-1291

2. Jaeggi T, Lussi A (2014) Prevalence, incidence and distribution of erosion. In: Lussi A, Ganss C (eds) Erosive tooth wear-from diagnosis to therapy, Monographs in Oral Science vol 25. Karger, Basel, pp 55-73. doi: 10.1159/000360973

3. Bartlett D, Lussi A, West N, Bouchard P, Sanz M, Bourgeois D (2013) Prevalence of tooth wear on buccal and lingual surfaces and possible risk factors in young European adults. J Dent 41:10071013. doi:10.1016/j.jdent.2013.08.018

4. Lussi A, Ganss C (eds) (2014) Erosive tooth wear-from diagnosis to therapy, 2nd edn, vol 25. Karger, Basel

5. Smith BG, Knight JK (1984) An index for measuring the wear of teeth. Br Dent J 156:435-438

6. Bartlett D, Ganss C, Lussi A (2008) Basic Erosive Wear Examination (BEWE): a new scoring system for scientific and clinical needs. Clin Oral Investig 12(Suppl 1):S65-S68. doi:10.1007/ s00784-007-0181-5

7. Salas MM, Nascimento GG, Vargas-Ferreira F, Tarquinio SB, Huysmans MC, Demarco FF (2015) Diet influenced tooth erosion prevalence in children and adolescents: results of a meta-analysis and meta-regression. J Dent. doi:10.1016/j.jdent.2015.05.012 